\title{
Analog Least Mean Square Adaptive Filtering for Self-Interference Cancellation in Full-Duplex Radios
}

\author{
Anh Tuyen Le, Le Chung Tran, Xiaojing Huang, Y. Jay Guo, and Lajos Hanzo
}

\begin{abstract}
In-band full-duplex (IBFD) radio represents one of the key technologies for future wireless communication and radar applications. A major challenge of this technology is to mitigate the strong self-interference (SI), so that the residual SI level falls below the receivers noise floor. Radio frequency (RF) selfinterference cancellation (SIC) is essential for preventing an IBFD receiver from becoming saturated by the SI. We commence with an in-depth review of the promising analog least mean square (ALMS) adaptive filtering architecture, conceived for RF SIC in the IBFD radio RF frontend. The cancellation circuits employing this architecture can be implemented purely by analog components without any involvement of more power-thirsty digital signal processing (DSP). The behaviours, performance and implementation of the ALMS loop are presented. Finally, their applications in various IBFD radios are discussed and future research directions are provided.
\end{abstract}

Index Terms-Least mean square algorithm, in-band fullduplex, self-interference cancellation, OFDM, MIMO, I/Q imbalance, Synthetic Aperture Radar.

\section{INTRODUCTION}

$\mathbf{F}$ OR many decades, wireless transceivers have operated in a half-duplex mode relying on two separate frequency bands at the same time or on two different time slots in the same frequency band for transmission and reception, respectively. With the number of wireless systems and networks soaring, it becomes imperative to find more efficient ways of exploiting the scarce frequency resources. In-band full-duplex (IBFD) radio enables the transceivers simultaneously transmit and receive signals in the same frequency band, which is widely regarded as a promising solution. Unfortunately, moving from half-duplex to IBFD systems is very challenging due to the self-interference (SI) imposed, since a strong transmitted signal would saturate the co-located receiver, contaminating the weak received signal. Hence, self-interference cancellation (SIC) is of fundamental importance to make the IBFD system feasible.

The SI is typically mitigated in three consecutive stages, namely, by separating the transmit $(\mathrm{Tx})$ and receive $(\mathrm{Rx})$ antennas, by analog domain (i.e., the RF stage) cancellation, and by digital domain cancellation. The analog domain cancellation is particularly critical to prevent the analog-todigital converter (ADC) from becoming saturated. However, implementing SIC in the analog domain is very challenging because the SI is unknown to the receiver even though the baseband equivalent of the transmitted signal is known. The reason is that, once the known digital signal is converted

Anh Tuyen Le, Xiaojing Huang and Y. Jay Guo are with the University of Technology Sydney. Le Chung Tran is with the University of Wollongong. Lajos Hanzo is with the University of Southampton. to the RF signal and emitted by the antenna, the analog SI becomes unknown because of the non-linearity of the RF components in the transmitter RF chain and owing to the reflections by surrounding objects. One must synthesize the transmitted analog signal to cancel the SI at the RF frontend of the receiver. Hence the cancellation circuit acts as an adaptive filter (AF) controlled by minimizing the difference between the SI and the synthesized cancellation signal.

RF cancellers can be further categorized as digital and analog AFs. Naturally, digital AFs employ digital signal processing (DSP) to calculate the weighting coefficients of the $\mathrm{AF}$ in order to minimize the error between the cancellation signal and the SI. Therefore, down-converters and ADCs are required to digitize the residual SI, as in [1]-[3]. The digital weighting coefficients are then converted to their analog counterparts by additional digital-to-analog converters (DAC) before modifying the delayed transmitted signal at a vector modulator. The digitized residual SI can also be captured after the ADC of the main receiver chain to estimate the SI channel. The digital transmitted signals are convolved with the estimated SI channel impulse response (CIR) to generate the baseband cancellation signal, which is then up-converted and amplified by an additional Tx chain [4], [5] to produce the RF cancellation signal.

By contrast, analog AFs do not require DSP for calculating their weighting coefficients. Instead, adaptive algorithms, such as the continuous-time analog-domain least mean square (LMS) method relying on an integrator are implemented using analog devices. However, since using an ideal integrator is impractical in the RF domain, implementing an analog version of the LMS algorithm becomes a challenge. As a result, first down-converters are employed to shift the transmitted RF signal and the residual SI to a lower frequency band. Then, analog multipliers and integrators [6] or low-pass filters [7] are utilized for synthesizing the analog weighting coefficients, which are used for further processing the delayed transmitted signal by a vector modulator. These extra down-conversion, multiplication and integrator modules make the conventional AFs costly in practical systems.

To conceive a solution for adaptive filtering directly at the $\mathrm{RF}$, a modified version of the weighting coefficient update method is proposed in [8], which uses a first-order LPF to replace the ideal integrator. This purely analog LMS AF architecture is termed as an analog LMS (ALMS) loop, which is illustrated in Fig. 1, where an $L$ tap AF cancels the SI impinging on the $\mathrm{Rx}$ antenna. The transmitted signal at the input of the Tx antenna after the high power amplifier (HPA) is used as the reference signal having the power of 
TABLE I

COMPARISON OF ALMS LOOP AND OTHER AFS FOR RF SIC

\begin{tabular}{|l|c|c|c|c|c|}
\hline & {$[1],[2]$} & {$[3]$} & {$[4],[5]$} & {$[6],[7]$} & ALMS Loop [8-12] \\
\hline No DSP involvement & & & & $\checkmark$ & $\checkmark$ \\
\hline No additional Tx chain & $\checkmark$ & $\checkmark$ & & $\checkmark$ & $\checkmark$ \\
\hline No DAC or ADC & & & & $\checkmark$ & $\checkmark$ \\
\hline No channel state information (CSI) requirement & $\checkmark$ & $\checkmark$ & & $\checkmark$ & $\checkmark$ \\
\hline No additional down-converter & & & $\checkmark$ & & $\checkmark$ \\
\hline Sufficient SIC demonstrated & & $\checkmark$ & $\checkmark$ & & $\checkmark$ \\
\hline
\end{tabular}

$\frac{1}{2} V_{X}^{2}$, where $V_{X}$ is the root-mean-square (RMS) value of the baseband Tx signal. Each tap of the multi-tap filter of Fig. 1 is connected to the delay unit $T_{d}$, and an in-phase/quadrature (I/Q) demodulator is used for generating the product of the error signal with the delayed reference signal, followed by a pair of LPFs to update the complex weighting coefficient which, in turn, modifies the delayed reference signal at the I/Q modulator. The synthesized cancellation signal is obtained by combining the outputs of all the taps. Although, the weighting coefficients are obtained by correlating the Tx signal and the error signal which includes the desired signal received from the far-end, they are independent of the desired signal as the Tx signal is. Therefore, the cancellation signal is not affected by the desired signal, which has been experimentally confirmed in other analog-based AFs, such as that of [6]. After cancellation, the residual SI is amplified by the low noise amplifier (LNA) having a gain of $2 \mu$ and looped back to the input of all the taps. Assuming that the the multipliers of the demodulator and modulator in each tap have dimensional constants of $K_{1}$ and $K_{2}$, respectively, the loop gain is $\mu A^{2}$ where $A^{2}=\frac{V_{X}^{2}}{K_{1} K_{2}}$. It is worth noting that, the LNA contributes to the loop gain of the ALMS loop so that the higher gain of the LNA, the higher level of cancellation can be achieved by the loop.

Both digital and analog AFs have their respective pros and cons. In particular, digital AFs benefit from the more flexible control of the weighting coefficients compared to their analog counterparts. However, the involvement of DSP for the associated weighting coefficient updates requires additional modules, such as down-converters and ADCs/DACs [1]-[3] which create unavoidable quantization noise. The secondary Tx chains of [4], [5] are also necessary, which prevents the digital AFs from capturing the non-linearity of the main Tx chain if it exists. More importantly, the digital AFs will be prohibitively complex if they are used in multiple input multiple output (MIMO) IBFD systems, since much larger field programmable gate array (FPGA) and higher power consumption will be required. By contrast, analog AFs do not have any quantization noise or FPGA resource problem and can be readily implemented as application specific integrated circuits (ASICs), making low-cost MIMO IBFD systems attractive. From this perspective, analog AFs are preferred to their digital counterparts. Table I explicitly summarizes the ALMS loop's advantages over other RF AF structures found in the literature. Clearly, the ALMS loop requires less extra modules than other AFs, while providing a sufficiently high level of SIC

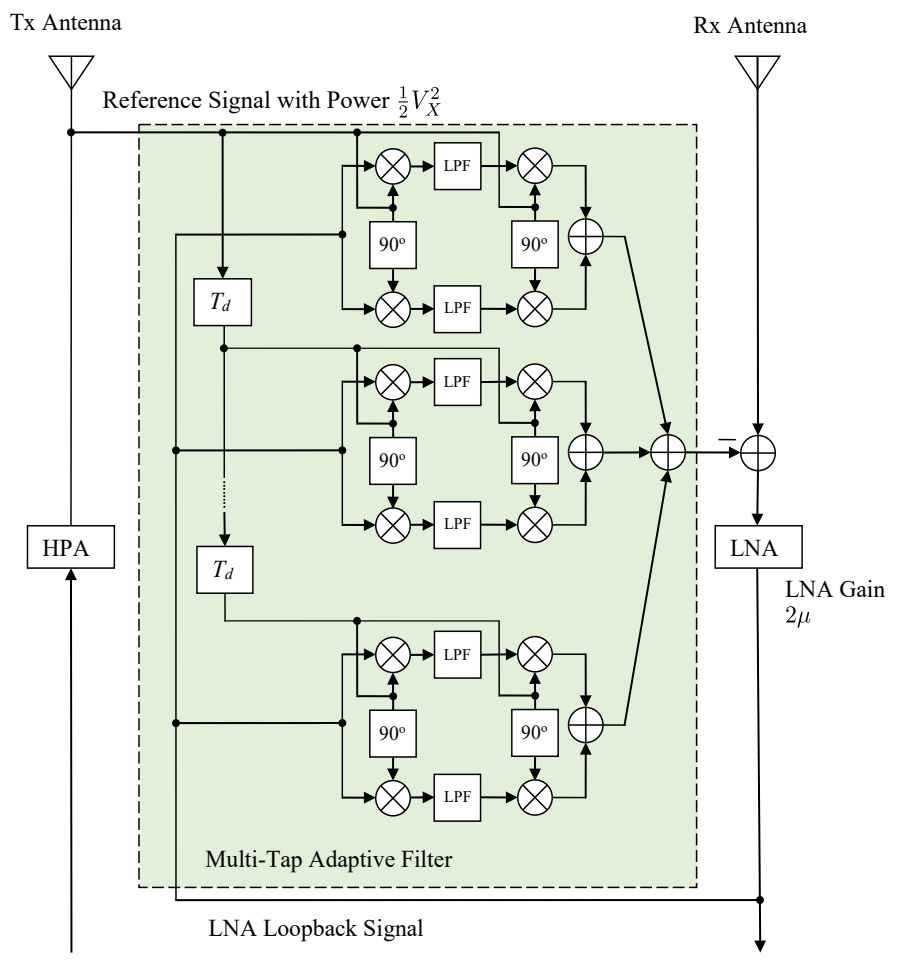

From Up-Converter

To Down-Converter

Fig. 1. ALMS loop with multi-tap adaptive filter

for satisfying the requirements of the RF stage (about $40 \mathrm{~dB}$ in practice).

Following the pioneering work on ALMS loops used in single carrier systems [8], further research has been conducted for investigating their behaviour in multi-carrier systems [9]. The frequency domain characteristics of the ALMS loop were then revealed, and the SIC performance bounds in both analog and digital domains were provided in [10]. Practical imperfections of the ALMS loop, such as its I/Q imbalance in the I/Q modulators and demodulators, on its performance were analyzed in [11]. An experimental prototype was also developed for verifying the theoretical results and for demonstrating the ALMS loops potential for efficient SIC [12].

In the rest of this article, detailed discussions on the ALMS loops behaviours at both micro-scale and macro-scale are provided. The performance bounds under various system parameters are presented in both analog and digital domains. The resilience of the ALMS loop to I/Q imbalance is charac- 
terised, followed by our experimental measurement results for quantifying the ALMS loops performance. The applications of the ALMS AFs in IBFD MIMO radios relying on the beam-based SIC structure [13] are presented. In particular, a novel Tx beamforming technique is introduced for the IBFD MIMO radios to explore both propagation and analog domain SIC capabilities and achieve optimized communication performance. Additionally, the application of the ALMS loop in advanced imaging radar systems is also presented. Finally, future research directions are discussed, followed by our conclusions. Note that, unless otherwise stated, the following parameters are used to obtain the simulation results. The IBFD system operates at the carrier frequency of $2.4 \mathrm{GHz}$ with a data symbol duration $T_{s}=65 \mathrm{~ns}$ and roll-off factor of $\beta=0.25$. The ALMS loop includes 8 taps and the tap delay $T_{d}=T_{s} / 2$. The transmitter and receiver use separate antennas and the propagation loss is $25 \mathrm{~dB}$.

\section{Micro- AND MACRO-SCALE Behaviour of ALMS LOOPS}

A significant difference between digital and analog adaptive filters is that the latter deals with continuous RF signals. At the micro-scale, we consider the cyclostationarity of the transmitted signal to examine the loops behaviour on a timescale of several data symbols. As revealed in [8], this cyclostationarity prevents the weighting coefficients of the ALMS loop from converging to stable values. The variation range of the weighting coefficients is determined by the transmitted signals properties, the loop gain and the LPF parameters. A generalized solution to determine the weighting coefficient variations for arbitrary cyclostationary transmitted signals was provided in [9], indicating that owing to this variation, the ALMS loop is unable to completely cancel the SI. In other words, there will be an irreducible residual SI, hence the normalized residual SI (NRSI), defined as the average residual SI power after cancellation versus the average SI power without cancellation, exhibits an lower bound. The NRSI lower bounds (NRSILBs) for both single carrier and orthogonal frequency division multiplexing (OFDM) signals were derived in [8] and [9], respectively. It has been shown in [9] that the NRSILBs can be determined by the decay constant $\alpha$ of the LPF used in the ALMS loop and by the transmitted signal properties including the data symbol duration (sampling period) $T_{s}$ and the roll-off factor $\beta\left(\beta_{o}\right)$ of the pulse shaping filter (windowing function) of the single carrier system (OFDM system), respectively. For an appropriately selected constant $\alpha T_{s}<5 \times 10^{-5}$, the NRSILB in both systems can be below $-100 \mathrm{~dB}$. It is also worth noting that the constant $\alpha T_{s}$ determines the convergence speed of the ALMS loop [8], [9]. A smaller $\alpha T_{s}$ decreases the weighting coefficient variation and hence reduces the NRSILB, but results in an increased convergence time.

By contrast, at the macro-scale, i.e., upon treating the transmitted signal as a stationary process to assess the overall loop performance, the ALMS loop performance is ultimately determined by the loop gain $\mu A^{2}$, the transmitted signals spectral properties, and the interference channel modeling error of the adaptive filter, which is related to the time delay

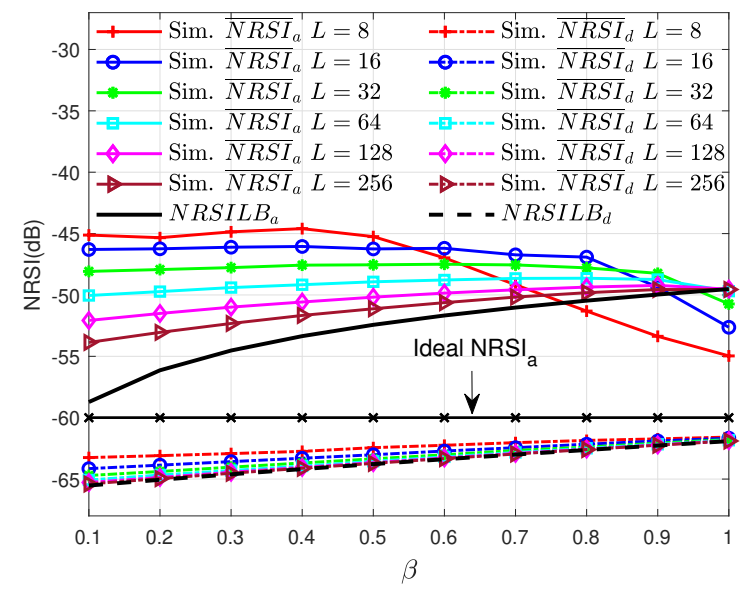

Fig. 2. NRSI versus roll-off factor $\beta$ in analog and digital domains for different number of taps in ALMS loop with $\mu A^{2}=1000$ and $T_{d}=T_{s} / 2$.

between a pair of adjacent taps and the total number of taps as determined by the channel impulse response duration. Ignoring any practical imperfection including the interference channel modeling error and after the ALMS loops output has stabilized, the ideal NRSI can be expressed as $\left(1+\mu A^{2}\right)^{-2}$. Therefore, as long as a sufficiently high loop gain is provided, the ALMS loop achieves a substantial SIC, typically about 40 to $60 \mathrm{~dB}$ based on practical prototyping [12].

\section{Performance Bounds in the Analog And Digital DOMAINS}

Both the theoretical analysis and the simulation results relying on a real SI channel showed in [8] that a finer tap delay resolution results in a higher level of cancellation. However, neither the role of tap delay nor the number of taps on the performance of the ALMS loop was quantified in [8]. Therefore, the analysis of [10] addressed this issue by showing that the expected level of NRSI is determined by the loop gain, the delay between adjacent taps and the number of taps. Fundamentally, the AF of the ALMS loop has a multitap structure in which the time delay between adjacent taps should obey the sampling theorem. Specifically, each tap of the AF in the ALMS loop aims for mimicking a "sample" of the SI, and the time difference between two adjacent taps represents the sampling period. Therefore, by assuming that the number of taps is sufficiently large, the NRSI derived in the time domain denoted as $\overline{N R S I}_{a}$ can be transformed to the frequency domain for evaluation by eigenvalue decomposition. In this way, the frequency response of the ALMS loop can be determined, which shows that the ALMS loop tends to amplify the residual SI frequency components at the edges of the signal spectrum. However, the amplified frequency components can be then removed by the matched filter in the digital domain, leading to a significant reduction of the NRSI in the digital domain, denoted as $\overline{N R S I}_{d}$. Furthermore, we assumed ideal root raised-cosine pulse shaping as well as matched-filtering and approximated the sum of discrete spectral components involved in the evaluations of $\overline{N R S I}_{a}$ and $\overline{N R S I}_{d}$ by the 
integral of their respective continuous power spectra, when the number of taps tends to infinity. With these assumptions, the respective NRSI lower bounds, denoted by $N R S I L B_{a}$ and $N R S I L B_{d}$, can be readily found in both the analog and digital domains. These lower bounds are determined by the loop gain, the roll-off factor $\beta$ of the transmit pulse shaping filter and the tap delay. It means that for a wider bandwidth, the ALMS loop should have finer tap delay resolution and possibly larger number of taps, depending on the length of interference channel impulse response.

Fig. 2 shows the simulation results for a single carrier IBFD radio having an ALMS loop gain of $\mu A^{2}=1000$ and tap delay $T_{d}=T_{s} / 2$, compared to the respective lower bounds $N R S I L B_{a}$ and $N R S I L B_{d}$, which indicates that when more taps are employed in the ALMS loop, the NRSIs in both the analog and digital domains approach their lower bounds, respectively. Note that the ideal analog domain NRSI at a loop gain of $\mu A^{2}=1000$ would be $-60 \mathrm{~dB}$. After removing the enhanced frequency components in the digital domain, we even have further 2 to $5 \mathrm{~dB}$ reduction over the ideal NRSI in the analog domain.

The analyses presented in [8]-[10] assumed that the delays are perfectly identical $T_{d}$ among all the taps. However, it is possible that they are different. If the delay $T_{d}$ is much smaller than the symbol duration $T_{s}$, the resultant small amount of mismatch does not affect the performance of the ALMS loop, since the combination of "samples" created at all the taps can still successfully mimic the SI. However, in case $T_{d}$ is close or equal to $T_{s}$, the delay mismatch will lead to spectral overlapping, because the ALMS loop violates the Nyquist theorem. Hence, to avoid this problem, $T_{d}$ should be chosen as a fraction of $T_{s}$.

\section{IMPACT OF I/Q IMBALANCE ON THE ALMS LOOP PERFORMANCE}

Most analyses on ALMS performance have been conducted in the ideal scenario of having no practical imperfection in the ALMS loop. Since the ALMS loop is an I/Q based structure, its performance will be affected by the I/Q imbalance within the loop. This problem is analyzed in [11]. Let us assume that the phase shifter of Fig. 1 has phase and amplitude errors of $\theta$ and $\rho$, respectively. The I/Q imbalance implies that the amplitudes of the signals in each tap of the two branches are imbalanced. It is also shown in [11] that the amplitude error is the only reason for the loop gain variation. As a result, it can be readily compensated by adjusting the gain of the LNA for example. However, even though the loop gain has been calibrated to be the same as that in the ideal case, the level of cancellation may still be reduced due to the I/Q imbalance. By averaging over many realizations of the SI channel, the degradation was shown to be insignificant in [11] under frequency-independent I/Q imbalance. An upper bound of the degradation is also derived in [11], demonstrating that, even in the worst case of $\rho= \pm 3 \mathrm{~dB}$ and $\theta= \pm 5^{\circ}$, the maximum I/Q imbalance degradation is limited to about 3.5 $\mathrm{dB}$. This upper bound assists us in determining how much a suppression level is required in the other SI mitigation domains to compensate for the degradation in the analog domain, given a specific I/Q imbalance level in the ALMS loop.

Frequency-dependent I/Q imbalance may be encountered by the ALMS loop in wide-band applications and different levels of cancellation might be achieved across the entire bandwidth. However, the differences are expected to be still within the limit of 3-4 dB, as long as the I/Q imbalance levels encountered at all frequencies in the band are below $\rho= \pm 3 \mathrm{~dB}$ and $\theta= \pm 5^{\circ}$. Therefore, the degradation can be potentially compensated by increasing the overall loop gain and/or incorporating sophisticated cancellation techniques.

\section{HARDWARE VERIFICATION RELYING ON AN EXPERIMENTAL PROTOTYPE}

In order to confirm the theoretical analyses conducted in [8]-[11], a prototype of the ALMS loop has been built to obtain experimental results. Additionally, although the structure of the ALMS loop is relatively simple, its implementation is still a challenge, since no high-gain multiplier is available in practical RF bands at high carrier frequencies. These problems are tackled in [12] as follows. The pair of multipliers in each tap of the ALMS loop are replaced by a quadrature demodulator and a modulator, respectively. Since the modulator and demodulator have low gains, adjustable-gain amplifiers are added at both input ports, and one more amplifier is added after the power combiner at the output of the cancellation circuit. Another issue associated with the modulator and the demodulator is that some upper harmonics of the input frequency appear at their outputs. This problem is solved as follows. The harmonics at the output of the demodulator are removed by the LPF, while those of the modulator can be significantly attenuated by selecting the amplifier at the output of the ALMS loop to have an appropriate frequency response, which removes the harmonics. A 2-tap prototype was developed based on this practical structure to obtain some experimental results for different $\mathrm{Tx}$ bandwidths, different roll-off factors of the Tx pulse shaping filters, and different signaling schemes. The prototype employed the demodulator ADL5382 and the modulator ADL5373 at each tap of the loop and a 4 ns delay line IC for the tap delay. A Wilkinson power divider/combiner was used to split or combine the RF signals. The transmitted signal is generated by the arbitrary waveform generator Keysight M8190A. The residual SI signal after the LNA was monitored by a spectrum analyzer. It is reported in [12] that the cancellation levels achieved by the prototype in the single carrier IBFD settings having $20 \mathrm{MHz}$ and $50 \mathrm{MHz}$ bandwidths are about $39 \mathrm{~dB}$ and $33 \mathrm{~dB}$, respectively. These levels are justifiable based on the theoretical values calculated in [10] taking into consideration the I/Q imbalance analyzed in [11]. The experiments associated with different roll-off factors of the pulse shaping filter in single carrier and OFDM modems also validate the conclusions in [9]. Fig. 3 shows the measurement results for the single carrier case having 20 $\mathrm{MHz}$ bandwidth. Interested readers might like to refer to [12] for more details on the practical loop parameter calculations, the expected level of cancellation and other experiments for different bandwidths, OFDM signal configurations and roll-off factors $\beta$. 


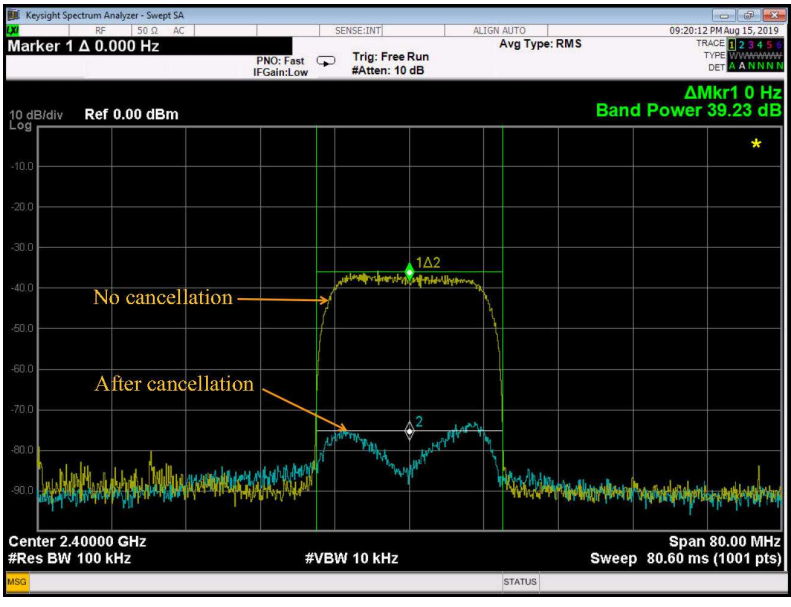

Fig. 3. Experimental results for SI cancellation in single carrier system with $20 \mathrm{MHz}$ bandwidth.

\section{APPLiCATIONS OF THE ALMS LOOP}

In addition to SIC in IBFD single-antenna systems, the ALMS loop can also be utilized in other applications, such as IBFD MIMO communication systems and full-duplex synthetic aperture radars.

\section{A. Application in IBFD MIMO Systems}

Naturally, the combination of IBFD technology and massive MIMO can further improve spectral efficiency. However, mitigating SI in IBFD MIMO systems is much more challenging because of the complex SI leakage channels. More specifically, in an $N \times N$ IBFD MIMO system, the SI within each Rx chain is a mixture of all the cross-talks emanating from $N \mathrm{Tx}$ antennas. Therefore, as stated in [14], if a cancellation circuit is used for each pair of Tx and Rx antennas, $N \times N$ cancellation circuits will be required for RF domain cancellation. In addition, if the cancellation circuit requires DSP to optimize its weights, the complexity of DSP algorithms also increases quadratically with the number of MIMO antennas due to the higher digital hardware cost of large FPGAs.

Since the ALMS loop does not require any DSP to tune its weights, it becomes a promising candidate for RF domain SIC in IBFD MIMO systems. The problem here is how to reduce the number of cancellation circuits, while the level of mitigation still remains the same. This issue is solved in [13] using beam-based analog SIC. In a massive MIMO system that transmits $K$ independent data streams, these $K$ streams are multiplied by a beamforming (BF) matrix before being upconverted by $N$ Tx chains and emitted through $N$ Tx antennas. Since many Tx chains are used, only a low transmit power per chain is necessary and hence the HPA can operate in its linear range. As a result, the SI imposed on each Rx chain can be seen as a linear transformation of the $K$ Tx beams. This means that the cancellation signal applied to each $\mathrm{Rx}$ chain should also be obtained by another linear transformation of the same $K$ Tx beams. Therefore, as few as $K$ ALMS loops are sufficient (instead of $N$ ) to cancel the SI at $M \mathrm{Rx}$ antennas. Mathematically, the beam-based SIC concept can be explained by the fact that the vector of SI signals at a single Rx chain is

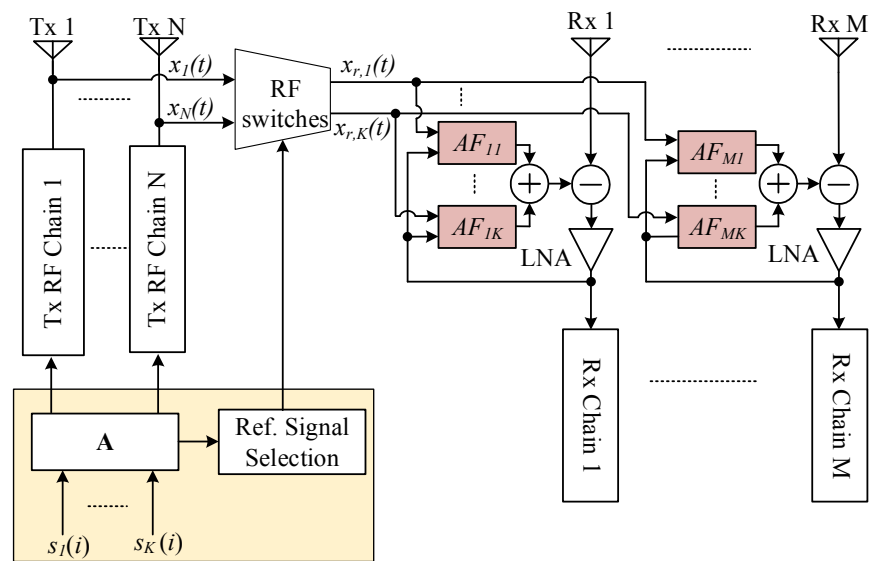

Fig. 4. Beam-based analog SI cancellation structure for MIMO systems making use of ALMS loops.

the sum of $N$ SI vectors, which are only generated in a space of $K$ dimensions spanned by the $K$ data streams. Hence, if the vector of cancellation signals is also constructed from the same space of $K$ dimensions, it can cancel the SI. Since $K$ is normally much smaller than the number of Tx antennas $N$, the number of AFs can be significantly reduced. Fig. 4 presents this beam-based SIC structure associated with a reference signal selection in which $K$ reference signals are selected from $N$ available Tx signals. An algorithm was developed in [13] to determine the sub-optimal reference signals so that the level of cancellation achieved by this structure can be even higher than that obtained by the same ALMS loop in a single-antenna system.

Another advantage of IBFD MIMO systems over their single-antenna counterpart is that since multiple antennas are available in the former, $\mathrm{BF}$ techniques can be used for reducing the SI at the co-located receiver. When the number of Tx antennas $N$ is larger than that of the Rx antennas $M$, the nullspace of the SI channels exists and a suitable Tx BF matrix (i.e., precoder) can be designed to null the interference at the receiver [15]. This is an ideal case where the SI channels can be perfectly estimated and sufficiently high-precision DSP is available in the digital baseband for calculating the Tx BF matrix. However, in practice, the level of SI suppression has to be traded off against the BF capability of the transmitter and receiver. In particular, the smaller $M$ is compared to $N$, the higher the level of SI suppression becomes at the receiver and the lower the precoding imperfection is at the transmitter. However, a smaller number of Rx antennas deteriorates the BF performance of the receiver. The beam-based analog cancellation using the ALMS loops can help to relax this trade-off by reducing the level of suppression required in the propagation domain. By combining this structure with the Tx BF technique and using higher sub-space dimensions for Tx BF, the number of Rx antennas can be increased to achieve improved MIMO communication capability, while the level of SIC can still be satisfied to ensure that the overall received signal level fits into the dynamic range of the ADCs.

Through further analyzing the performance of multibeam 


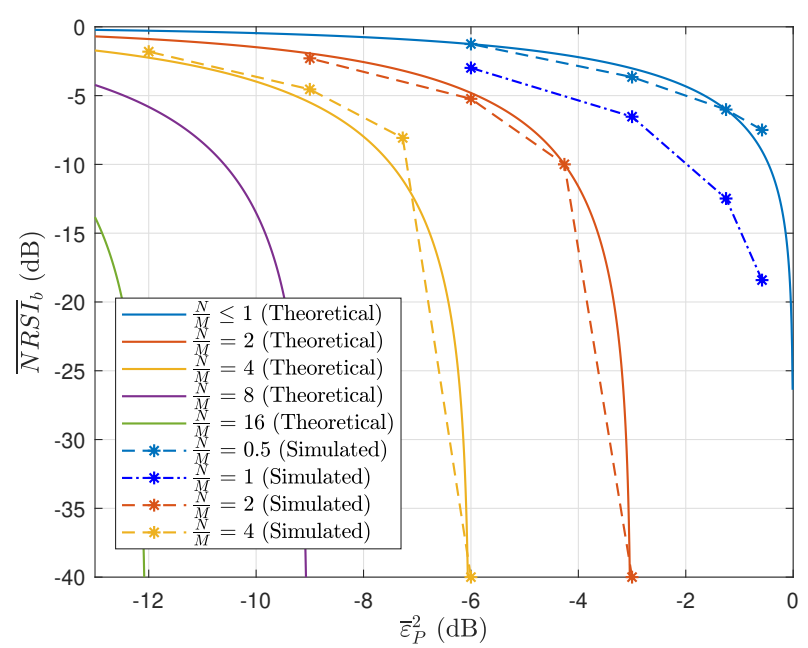

Fig. 5. Theoretical and simulated $\overline{N R S I}_{b}$ and precoding error trade-off curves.

communications using optimized Tx precoding and SIC-aided by Tx BF in an IBFD MIMO system, closed-form expressions of the NRSI and the Tx precoding error trade-off can be derived given the ratio between the numbers of $\mathrm{Tx}$ and $\mathrm{Rx}$ antennas. The formulated trade-off curves can provide guidelines for IBFD MIMO transceiver design. Fig. 5 shows the theoretical and simulated trade-off presented as a set of NRSIs attained by Tx BF, denoted as $\overline{N R S I}_{b}$, versus the Tx precoding error $\bar{\epsilon}_{P}^{2}$ curves recorded for various $N / M$ ratios. The theoretical $\overline{N R S I}_{b}$ is obtained as $\sigma_{H}^{2}+1-\bar{\varepsilon}_{P}^{2}$ for $N \leq M$, and $\sigma_{H}^{2}+\frac{\left(1-\frac{N}{M} \bar{\varepsilon}_{P}^{2}\right)^{2}}{1-\bar{\varepsilon}_{P}^{2}}$ for $N>M$, where $\sigma_{H}^{2}$ is the mean square error of channel estimation, and it is assumed to be $-40 \mathrm{~dB}$. To illustrate how the combined SIC using Tx BF and beambased ALMS loops relying on both the propagation and analog domains can make the selection of Rx antennas more flexible, let us first set $N=16$ and $M=4$. From the trade-off curve of $N / M=4$, the Tx BF can provide an $\overline{N R S I}_{b}$ of $-40 \mathrm{~dB}$ while the Tx precoding error is $\bar{\epsilon}_{P}^{2}=-6 \mathrm{~dB}$. Considering a $25 \mathrm{~dB}$ antenna isolation, the total NRSI over the propagation domain is $-25-40=-65 \mathrm{~dB}$ which is required to prevent signal clipping at the receiver's ADCs. To use more Rx antennas, say $M=8$, the NRSI of Tx BF can only be $-5 \mathrm{~dB}$ while the Tx precoding error is still maintained at $-6 \mathrm{~dB}$ according to the trade-off curve of $N / M=2$. The propagation-domain NRSI will only be $-25-5=-30 \mathrm{~dB}$. However, assuming that the beam-based ALMS loops in the analog domain can provide an SI suppression of $39 \mathrm{~dB}$ by appropriately selecting the loop gain and other parameters, the total NRSI will be $-69 \mathrm{~dB}$, still satisfying the ADC requirement.

\section{B. Application in Full-duplex Synthetic Aperture Radar}

The combination of a full-duplex radio technique and a continuous wave synthetic aperture radar (SAR), called generalized continuous wave synthetic aperture radar (GCW-SAR), has been developed recently. In such systems, the slow time sampling in azimuth direction used in a conventional pulsed SAR system is no longer required, thus the minimum antenna aperture constraint is eliminated. This new SAR concept enables the development of future high-resolution and wideswath remote-sensing technologies. To achieve this compelling advantage, cancelling SI is also a key problem.

Self-interference cancellation in GCW-SAR systems is rather different from that in IBFD systems. In particular, RF SIC in GCW-SAR applications is very important, since it can make digital domain cancellation unnecessary for reducing the imaging processing complexity. The reason is that, as long as the ADC is not saturated, digitized SI can be considered as a near-field reflection, which is readily distinguishable from the far-field reflections of the target. Additionally, a deterministic chirp signal is transmitted at the GCW-SAR system, in contrast to the random information bearing signal at the IBFD communication system. This difference in the transmitted signal may affect the convergence properties of the ALMS loop. Specifically, the periodical transmitted signal of GCW-SAR has a periodical autocorrelation function whereas the average autocorrelation function of the random transmitted signal for data communications is aperiodic. Hence, the number of taps in the loop must be constrained so that only one period of the chirp signals autocorrelation function is involved in updating the weighting coefficients. Furthermore, the tap delay must be determined by the bandwidth of the chirp signal to avoid spectral overlapping. When the ALMS loop meets these constrains, it performs well together with propagation domain suppression without the need for digital cancellation.

\section{Future Research Directions on ALMS Loop}

Throughout the above-mentioned studies, a complete picture of the ALMS loop has been drawn from different perspectives. Future research ideas include the development of IBFD solutions and the extension of the ALMS loop to other applications.

To develop a complete IBFD system, several research problems will have to be tackled. Firstly, the noise resilience of the ALMS loop should be investigated. In particular, since some non-linear components such as the modulator and demodulator are involved in the loop, their impact on the receiver noise level should also be considered. Moreover, since the weighting coefficients of the ALMS loop are obtained by correlating the delayed transmitted signal and the error signal that is fed back, they might be affected by the signal of interest. As a result, some additional noise will contaminate the residual signal. Therefore, a joint digital cancellation amalgamated with an appropriately designed transmission protocol should be developed for minimizing the impact on the receiver's sensitivity. Secondly, to reduce the SI power below the noise floor, a combination of the ALMS loop with realistic propagation-domain and digital-domain solutions should also be studied. To evaluate the performance of the overall threestage cancellation, the signal to noise and interference ratio at a complete IBFD receiver should be analyzed. Finally, to minimize the physical size and make the ALMS loop suitable for low-power applications, an integrated circuit based ALMS loop should be developed.

Another research direction is to adopt the ALMS loop for other applications, which require adaptive signal processing 
in the RF frontend. As an example, the ALMS loop may be used in military applications to protect a receiver from electronic attacks. With the ALMS loop at the RF frontend, the jamming signal will be cancelled, so that the transceiver can still communicate with its far-end receiver. Additionally, the weighting coefficients within the loop are related to the CSI so that it can be used to estimate the location of the attacker. However, in these cases, the structure of the ALMS loop may be adjusted to suit the specific target requirements.

\section{CONCLUSIONS}

This article reviewed ALMS adaptive filtering aided RF domain SIC. The lack of ideal RF integrators leads to the development of the ALMS loop, which employs simple resistorcapacitor LPFs to replace the ideal integrators. The performance of the ALMS loop and the impacts of I/Q imbalance on SIC performance were presented for different transmitted signals. A practical prototype of the ALMS loop using offthe-shelf components was also described and experimental results were provided to confirm the theoretical findings. Beneficial applications of the ALMS loop in different IBFD radios, such as IBFD MIMO transceivers and GCW-SAR RF solutions, were also given. These findings were confirmed by our experimental results, indicating that the ALMS loop is a promising RF SIC solution.

\section{ACKNOWLEDGMENT}

This work is supported by the Australian Research Council Discovery Project DP200101532.

L. Hanzo would like to acknowledge the financial support of the Engineering and Physical Sciences Research Council projects EP/N004558/1, EP/P034284/1, EP/P034284/1, EP/P003990/1 (COALESCE), of the Royal Society's Global Challenges Research Fund Grant as well as of the European Research Council's Advanced Fellow Grant QuantCom.

\section{REFERENCES}

[1] K. E. Kolodziej, J. G. McMichael, and B. T. Perry, "Multitap RF canceller for in-band full-duplex wireless communications," IEEE Trans. Wireless Commun., vol. 15, no. 6, pp. 4321-4334, June 2016.

[2] I. Hwang, B. Song, C. Nguyen, and S. S. Soliman, "Digitally controlled analog wideband interference cancellation for in-device spectrum sharing and aggregation," IEEE J. Sel. Areas Commun., vol. 34, no. 11, pp. 2838-2850, 2016.

[3] D. Korpi, J. Tamminen, M. Turunen, T. Huusari et al., "Full-duplex mobile device: Pushing the limits," IEEE Commun. Mag., vol. 54, no. 9, pp. 80-87, Sept. 2016.

[4] F. J. Soriano-Irigaray, J. S. Fernandez-Prat, F. J. Lopez-Martinez, E. Martos-Naya et al., "Adaptive self-interference cancellation for full duplex radio: Analytical model and experimental validation," IEEE Access, vol. 6, pp. $65018-65026,2018$.

[5] A. Kiayani, M. Z. Waheed, L. Anttila, M. Abdelaziz et al., "Adaptive nonlinear RF cancellation for improved isolation in simultaneous transmit-receive systems," IEEE Trans. Microw. Theory Techn., vol. 66, no. 5, pp. 2299-2312, Jan. 2018.

[6] T. Huusari, Y. S. Choi, P. Liikkanen, D. Korpi et al., "Wideband selfadaptive RF cancellation circuit for full-duplex radio: operating principle and measurements," in Proc. 81st IEEE Veh. Technol. Conf. (VTC Spring), June 2015, pp. 11-14.

[7] G. Noh, H. Wang, C. Shin, S. Kim et al., "Enabling technologies toward fully LTE-compatible full-duplex radio," IEEE Commun. Mag., vol. 55, no. 3, pp. 188-195, 2017.
[8] X. Huang and Y. J. Guo, "Radio frequency self-interference cancellation with analog least mean-square loop," IEEE Trans. Microw. Theory Tech. vol. 65, no. 9, pp. 3336-3350, Sept. 2017.

[9] A. T. Le, L. C. Tran, and X. Huang, "Cyclostationary analysis of analog least mean square loop for self-interference cancellation in in-band fullduplex systems," IEEE Commun. Lett., vol. 21, no. 12, pp. 2738-2741, Sept. 2017.

[10] A. T. Le, L. C. Tran, X. Huang, Y. J. Guo, and J. C. Vardaxoglou, "Frequency domain characterization and performance bounds of ALMS loop for RF self-interference cancellation," IEEE Trans. Commun., vol. 67, no. 1, pp. 682-692, Jan. 2019.

[11] A. T. Le, L. C. Tran, X. Huang, and Y. J. Guo, "Analog least mean square loop with I/Q imbalance for self-interference cancellation in full-duplex radios," IEEE Trans. Vehicular Technol., vol. 68, no. 10, pp. $9848-$ 9860, Oct 2019.

[12] A. T. Le, L. C. Tran, X. Huang, and Y. J. Guo, "Analog least mean square loop for self-interference cancellation: A practical perspective," Sensors (Switzerland), vol. 20, no. 270, pp. 1-15, 2020.

[13] A. T. Le, L. C. Tran, X. Huang, and Y. J. Guo, "Beam-based analog selfinterference cancellation in full-duplex MIMO systems," IEEE Trans. Wireless Commun., vol. 19, no. 4, pp. 2460-2471, Jan. 2020.

[14] D. Bharadia and S. Katti, "Full duplex MIMO radios," in Proc. 11th USENIX Conf. Networked Systems Design Implementation, ser NSDI'14. Berkeley, CA, USA: USENIX Association, 2014, pp. 359372.

[15] E. Everett, C. Shepard, L. Zhong, and A. Sabharwal, "Softnull: Manyantenna full-duplex wireless via digital beamforming," IEEE Trans. Wireless Commun., vol. 15, no. 12, pp. 8077-8092, Dec. 2016.

\section{BIOGRAPHIES}

Anh Tuyen Le received his Ph.D. degree from University of Technology Sydney (UTS), Australia, in August 2020. He is currently a research assistant at the Global Big Data Technologies Centre, UTS. His research interests include full-duplex radio, RF localization, and high-speed wireless communications.

Le Chung Tran [SM'18] received his Ph.D. degree from University of Wollongong (UOW), Australia, in 2006. He was awarded with the postdoctoral research fellowship from the Alexander von Humboldt $(\mathrm{AvH})$ foundation in 2006-2008 and the renewed research stay award from the AvH in 2015. $\mathrm{He}$ is currently the senior lecturer at School of Electrical, Computer and Telecommunications Engineering (SECTE), UOW. His research interests include 5G, wireless body area networks, localization and positioning. $\mathrm{He}$ is a fellow of the Humboldt foundation.

Xiaojing Huang [SM'11] received the B.Eng., M.Eng., and Ph.D. degrees in electronic engineering from Shanghai Jiao Tong University, Shanghai, China, in 1983, 1986, and 1989, respectively. He currently is a professor of information and communications technology in the School of Electrical and Data Engineering and the program leader for mobile sensing and communications in the Global Big Data Technologies Centre of the University of Technology Sydney, Australia.

Y. Jay Guo [F'14] received his Bachelor's and a Master's degrees from Xidian University in 1982 and 1984, respectively, and a Ph.D. degree from Xi' an Jiaotong University in 1987, all in China. Currently, he is a Distinguished Professor and the director of the Global Big Data Technologies Centre at UTS. Prior to this, he served as a research director at CSIRO for over nine years, and held senior leadership positions at Fujitsu, Siemens, and NEC in the United Kingdom. 
Lajos Hanzo [F'04] FREng, FIEEE, FIET, Fellow of EURASIP, DSc, received his degree in electronics in 1976 and his doctorate in 1983. He holds an honorary doctorate from the Technical University of Budapest (2009) and from the University of Edinburgh (2015). He is a member of the Hungarian Academy of Sciences and a former Editor-in-Chief of the IEEE Press. He is a Governor of both IEEE ComSoc and of VTS and published 1900+ contributions at IEEE Xplore. 